\title{
Cardiac Arrhythmia Associated with Remdesivir in COVID-19
}

\author{
Sristee Niraula, MD*, Shital Oli, MD and Janette Lee, MD
}

Department of Internal Medicine, Cayuga Medical Center, USA

*Corresponding author: Sristee Niraula, Department of Internal Medicine, Cayuga Medical Center, NY, USA

\begin{abstract}
During the pandemic, Remdesivir was approved as a treatment for severe COVID-19. Little is known about its adverse side effects, as it had not been in clinical use prior to the pandemic, and we continue to learn more daily about its impact-both positive and negative-on our patients. In this case report, we present a patient who developed sinus bradycardia on the third day of Remdesivir administration, NSVT on the fifth day of Remdesivir, and whose arrhythmias resolved after cessation of Remdesivir.
\end{abstract}

\section{Introduction}

COVID-19 emerged to an unsuspecting world in late 2019 and by early 2020 the disease was so widespread that it was declared a pandemic by the World Health Organization. As our collective experience with, and understanding of this virus increases, guidelines for prevention and recommendations for treatment change constantly. For example, since the start of the pandemic, there have been multiple modifications in quarantine guidelines. Currently, the CDC recommends that asymptomatic patients quarantine for 10 days; there are, however, a few reports of infected patients who manifest symptoms after the quarantine period [1]. Different treatment options have also been touted as promising and later shown to be ineffective or harmful, leading clinicians to make decisions based on best-available evidence, and to be alert to unexpected events or outcomes. Remdesivir is one such medication that has been used extensively for severe symptoms [2] but whose true influence on outcome and potential for side effects is yet unclear. While common side effects reported with Remdesivir include GI symptoms, there have been a few case reports of cardiac complications with Remdesivir use [3]. Here, we report a case of an elderly male who developed symptoms 11 days after his positive COVID test, and who experienced arrhythmias while on Remdesivir.

\section{History of Present IIIness}

An 88-year-old male with a medical history significant for diabetes, hypertension and remote history of prostate and bladder cancer presented with generalized weakness and shakiness.

The patient incidentally tested positive for COVID-19 on December 26, 2020 and was asymptomatic throughout his quarantine at home. He was released from quarantine by the health department after 10 days. However, his symptoms of weakness and shakiness began on the $11^{\text {th }}$ day. He otherwise reported no chest pain, shortness of breath, nausea, vomiting, diarrhoea, loss of taste/smell, or abdominal pain.

On presentation to the Emergency Department, he was febrile with a temperature of $101.8^{\circ} \mathrm{F}$, with other vitals within normal limits and an oxygen saturation of $96 \%$ on room air. Physical exam was unremarkable.

\section{Past Medical History}

His past medical history included type 2 diabetes, hypertension and history of bladder and prostate cancer.

\section{Investigations}

Pertinent laboratory studies showed ferritin of $1527.7 \mathrm{ng} / \mathrm{ml}$, LDH of $281 \mathrm{U} / \mathrm{L}$, D-dimer of $328 \mathrm{ng} / \mathrm{ml}$ and lymphocyte count of 0.8 . His creatinine was 1.95 $\mathrm{mg} / \mathrm{dl}$. Other blood work was unremarkable including liver function tests and troponin.

Chest X-ray did not show any radiographic evidence for acute cardiopulmonary abnormalities. Due to his 
weakness, brain MRI and CT scan were done, both of which were normal. Abdomen/pelvis $\mathrm{CT}$ was negative for acute findings. Chest CT revealed extensive patchy, bilateral ground-glass opacities, more pronounced in the left lung. He was re-tested for COVID-19 and was positive. He was placed on a regimen of Remdesivir and Dexamethasone, along with Vitamin C and Zinc. During the course of treatment, the patient's heart rate was initially around $70-80$, but after day 3 , his heart rate dropped to the range of 50-60. On Day 5 of Remdesivir initiation, he demonstrated PVCs with accelerated wide complex tachycardia, the longest episode being 16 beats with a ventricular rate of 100 beats-per-minute. He was completely asymptomatic during this episode, which occurred approximately 50 minutes after his fifth dose of Remdesivir. Transthoracic echocardiogram was done that showed Ejection Fraction of $60-65 \%$ with no regional wall motion abnormalities; troponin was also negative. Patient was noted to have had multiple stress tests in the past, all of which were negative. Given that he was asymptomatic, with no troponin elevation, no ischemic changes on EKG and no acute findings on echo, we did not pursue a stress test or catheterization. He was observed on telemetry, and no further episodes were noted. He did not receive Remdesivir after that episode, as he had completed his regimen. On day 8 his heart rate returned to his baseline, around 70-80 bpm. He was discharged after receiving physical therapy.

\section{Discussion}

Remdesivir is an antiviral medication that inhibits the SARS-CoV-2-RNA-dependent RNA polymerase which is necessary for viral replication. It is an adenosine nucleotide prodrug that is metabolized to the pharmacologically active nucleoside triphosphate metabolite after being distributed into cells. It has been approved by FDA for use in COVID-19 patient's adults that are hospitalized.

Little has been reported about the side effects of Remdesivir, particularly any cardiovascular effects.
There have been a few case reports about Remdesivirinduced bradycardia and QTc prolongation. Here we report both bradycardia and wide-complex tachycardia which might be associated with Remdesivir use, as this patient began his hospital stay with a normal heart rate, and was seen to return to his baseline heart rate with no further episodes of arrhythmia after cessation of Remdesivir.

\section{Conclusion}

Our patient developed sinus bradycardia and wide complex tachycardia during Remdesivir use. Appropriate caution and continuous EKG monitoring should be done in patients using Remdesivir, as its safety profile remains uncertain. Even closer surveillance for patients with pre-existing heart disease may be warranted when using Remdesivir. There remains the need for more high-quality evidence from randomized controlled trials that are presently underway. A final note is that our case calls into question the current recommendations to end quarantine after 10 days without symptoms, as this patient developed symptoms after 10 days and required hospitalization. Indeed, clinicians should remain on high alert as patients, particularly in the elderly population, come off quarantine following expert consensus or guidelines.

\section{Learning Objectives}

- Recognize side effects of Remdesivir.

- Discuss late presentation of COVID.

\section{References}

1. Bowser AD (2020) Some infected patients could show COVID-19 symptoms after quarantine. Ann Intern Med.

2. Beigel JH, Tomashek KM, Dodd LE, Mehta AK, Zingman BS, et al. (2020) Remdesivir for the treatment of Covid-19 final report. N Engl J Med 383: 1813-1826.

3. Gubitosa JC, Kakar P, Gerula C, Nossa H, Finkel D (2020) Marked sinus bradycardia associated with remdesivir in COVID-19: A case and literature review. JACC Case Rep 2: $2260-2264$. 